PROCEEDINGS OF THE

AMERICAN MATHEMATICAL SOCIETY

Volume 128, Number 3, Pages 643-646

$\mathrm{S}$ 0002-9939(99)05655-5

Article electronically published on October 25, 1999

\title{
EIGENVALUE COMPLETIONS BY AFFINE VARIETIES
}

\author{
JOACHIM ROSENTHAL AND XIAOCHANG WANG
}

(Communicated by John A. Burns)

\begin{abstract}
In this paper we provide new necessary and sufficient conditions
for a general class of eigenvalue completion problems.
\end{abstract}

\section{Preliminaries}

Let $\mathbb{F}$ be an algebraically closed field of characteristic zero. Let $M a t_{n \times n}$ be the space of all $n \times n$ matrices defined over the field $\mathbb{F}$. We will identify $M_{a t} t_{n \times n}$ with the vector space $\mathbb{F}^{n^{2}}$. Let $\mathcal{X} \subset M a t_{n \times n}$ be an affine variety. If $M \in M_{n \times n}$ is a particular matrix we will denote by $\sigma_{i}(M)$ the $i$-th elementary symmetric function in the eigenvalues of $M$, i.e. $\sigma_{i}(M)$ denotes up to sign the $i$-th coefficient of the characteristic polynomial of $M$.

In this note we will be interested in conditions on the variety $\mathcal{X}$ which guarantee that the morphism

$$
\chi: \mathcal{X} \longrightarrow \mathbb{F}^{n}, \quad X \longmapsto\left(\sigma_{1}(A+X), \ldots, \sigma_{n}(A+X)\right)
$$

is dominant for a particular matrix $A$. In other words we are interested under what conditions the image misses at most a proper algebraic subset, i.e. the image forms a generic subset of $\mathbb{F}^{n}$. This problem was treated in [HRW97] when $\mathcal{X}$ is a linear subspace of $M_{a \times n}$ and the base field $\mathbb{F}$ consists of the complex numbers. In this paper we generalize those results to the situation when $\mathcal{X}$ represents a general affine (irreducible) variety defined over $\mathbb{F}$.

Our study is motivated in part by an extensive literature on matrix completion problems and by several applications arising in the control literature. We refer to the research monograph [GKS95], which provides a good overview on the large linear algebra literature on matrix completions and to the survey articles [Byr89, RW97] for the connections to the control literature and further references.

\section{MAin RESUlt}

Theorem 2.1. The characteristic map $\chi$ introduced in (1.1) is dominant for a generic set of matrices $A \in M a t_{n \times n} \cong \mathbb{F}^{n^{2}}$ if and only if $\operatorname{dim} \mathcal{X} \geq n$ and the trace function $\operatorname{tr} \in \mathcal{O}(\mathcal{X})$ is not a constant.

Received by the editors March 4, 1997 and, in revised form, April 2, 1998.

2000 Mathematics Subject Classification. Primary 15A18; Secondary 93B60.

Key words and phrases. Eigenvalue completions, pole placement problems, dominant morphism theorem, inverse eigenvalue problems.

The first author was supported in part by NSF grant DMS-9400965.

The second author was supported in part by NSF grant DMS-9500594.

(C)1999 American Mathematical Society 
The stated conditions are obviously necessary. Our proof is mainly based on two propositions. The first one is a strong version of the Dominant Morphism Theorem. Our formulation is immediately deduced from [Bor91, Chapter AG, $\S 17$, Theorem 17.3].

Proposition 2.2 (Dominant Morphism Theorem). Let $\phi: \mathcal{X} \rightarrow \mathcal{Y}$ be a morphism of affine varieties. Then $\phi$ is dominant if and only if there is a smooth point $P \in \mathcal{X}$ having the property that $\phi(P)$ is smooth and the Jacobian $d \phi_{P}: T_{P}(\mathcal{X}) \rightarrow T_{\phi(P)}(\mathcal{Y})$ is surjective.

The second proposition which we will need in the proof of Theorem 2.1 is:

Proposition 2.3 ([HRW97]). Let $\mathcal{L} \subset$ Mat $_{n \times n}$ be a linear subspace of dimension $\geq n, \mathcal{L} \not \subset s l_{n}$ (i.e. $\mathcal{L}$ contains an element with nonzero trace). Let

$$
\pi(L)=\left(l_{11}, l_{22}, \ldots, l_{n n}\right)
$$

be the projection onto the diagonal entries. Then there exists an $S \in G l_{n}$ such that

$$
\pi\left(S \mathcal{L} S^{-1}\right)=\mathbb{F}^{n} .
$$

This proposition was formulated in [HRW97, Lemma 2.8] when the base field $\mathbb{F}$ consists of the complex numbers. The proof presented in [HRW97] only requires linearizations of rational functions and it is therefore valid mutatis mutandis for an arbitrary base field $\mathbb{F}$.

Proof of Theorem 2.1. As mentioned earlier it is enough to show the sufficiency of the stated conditions. It has been pointed out in [HRW97] that the characteristic map $\chi$ is dominant, respectively surjective, if and only if the trace map

$$
\psi: \mathcal{X} \longrightarrow \mathbb{F}^{n}, \quad X \longmapsto\left(\operatorname{tr}(A+X), \ldots, \operatorname{tr}(A+X)^{n}\right)
$$

is dominant, respectively surjective. This follows from the so-called Newton identities which express the elementary symmetric functions $\sigma_{i}(M)$ in terms of the power sum symmetric functions $\left\{\operatorname{tr}\left(M^{j}\right) \mid 1 \leq j \leq n\right\}$.

It is the strategy of our proof to show the existence of a smooth point $P \in \mathcal{X}$ which has the property that the Jacobian $d \psi_{P}$ is surjective for a generic set of matrices $A \in M a t_{n \times n}$. Since the range of the trace map $\psi$ is a smooth variety, the proof would be complete.

Let $Q \in \mathcal{X}$ be a smooth point and consider the polynomial function

$$
f(M):=\operatorname{tr}(M)-\operatorname{tr}(Q) \in \mathcal{O}\left(\text { Mat }_{n \times n}\right)=\mathbb{F}\left[x_{11}, \ldots, x_{n n}\right] .
$$

Let $\mathcal{H}$ be the linear hypersurface

$$
\mathcal{H}:=\left\{U \in \operatorname{Mat}_{n \times n} \mid f(U)=0\right\} .
$$

Since $Q \in \mathcal{H} \cap \mathcal{X}$, it follows by the affine dimension theorem that the dimension of $\mathcal{H} \cap \mathcal{X}$ is at least $\operatorname{dim} \mathcal{X}-1$. Since by assumption $\mathcal{X}$ is irreducible and $\operatorname{tr} \in \mathcal{O}(\mathcal{X})$ is not a constant, it follows that

$$
\operatorname{dim}(\mathcal{H} \cap \mathcal{X})=\operatorname{dim} \mathcal{X}-1 .
$$

Let $\mathcal{S} \subset \mathcal{X}$ be the singular locus and let $\mathcal{I} \subseteq \mathcal{H} \cap \mathcal{X}$ be the irreducible component of $\mathcal{H} \cap \mathcal{X}$ which contains the smooth point $Q$. It follows that $\mathcal{S} \cap \mathcal{I}$ is a proper algebraic subset of $\mathcal{I}$. Because of this there exists a point $P \in \mathcal{I} \subset \mathcal{X}$ which is both smooth inside $\mathcal{I}$ as well as inside $\mathcal{X}$. 
By construction the tangent space $T_{P}(\mathcal{I})$ is properly contained inside the tangent space $T_{P}(\mathcal{X})$ and one has the relation

$$
T_{P}(\mathcal{I})=T_{P}(\mathcal{X}) \cap s l_{n}
$$

By Proposition 2.3 there exists an $S \in G l_{n}$ such that

$$
\pi\left(S\left(T_{P}(\mathcal{X})\right) S^{-1}\right)=\mathbb{F}^{n} .
$$

Consider the trace map $\psi$ introduced in (2.1). A direct computation shows that the Jacobian at the point $P$ is given through:

$$
d \psi_{P}: T_{P}(\mathcal{X}) \longrightarrow \mathbb{F}^{n}, \quad L \longmapsto\left(\operatorname{tr}(L), 2 \operatorname{tr}((A+P) L), \ldots, n \cdot \operatorname{tr}\left((A+P)^{n-1} L\right)\right) .
$$

Since the characteristic of $\mathbb{F}$ is zero, $\mathbb{F}$ contains as a prime field the rational numbers $\mathbb{Q}$. The matrices

$$
D:=\left(\begin{array}{cccc}
1 & & & \\
& 2 & & \\
& & \ddots & \\
& & & n
\end{array}\right), \quad V:=\left(\begin{array}{cccc}
1 & 1 & \cdots & 1 \\
1 & 2 & \cdots & 2^{n-1} \\
\vdots & \vdots & & \vdots \\
1 & n & \cdots & n^{n-1}
\end{array}\right)
$$

are therefore invertible. Define $A:=S^{-1} D S-P$. With our choice of the matrix $A$ the Jacobian is given through:

$$
\begin{aligned}
d \psi_{P}(L) & =\left(\operatorname{tr}\left(S L S^{-1}\right), 2 \operatorname{tr}\left(S L S^{-1} D\right), \ldots, n \operatorname{tr}\left(S L S^{-1} D^{n-1}\right)\right) \\
& =\pi\left(S L S^{-1}\right) V D .
\end{aligned}
$$

Since both the matrices $V$ and $D$ describe invertible transformations on $\mathbb{F}^{n}$, it follows that $d \psi_{P}$ is surjective for the particular choice of the matrix $A$. By the Dominant Morphism Theorem 2.2, $\psi$ and therefore $\chi$ is dominant.

Since the set of matrices $A$ whose associated Jacobian $d \psi_{P}$ forms a Zariski open set, and since we just showed that it is nonempty, it follows that for a generic set of matrices the map $\chi$ is dominant.

In the remainder of the paper we assume that $\mathcal{X} \subset \mathbb{F}^{n^{2}}$ is a fixed affine variety of dimension $\operatorname{dim} \mathcal{X}=m \geq n$, with coordinate $\operatorname{ring} \mathcal{O}(\mathcal{X})$ and vanishing ideal $I(\mathcal{X})$. We conclude the paper with an algebraic description of all matrices $A$ whose characteristic map is dominant.

Let $\left(f_{1}(X), \ldots, f_{k}(X)\right)$ be generators of $I(\mathcal{X})$ and define

$$
T(X)=\left(\begin{array}{ccc}
\frac{\partial f_{1}(X)}{\partial x_{11}} & \ldots & \frac{\partial f_{k}(X)}{\partial x_{11}} \\
\vdots & & \vdots \\
\frac{\partial f_{1}(X)}{\partial x_{1 n}} & \ldots & \frac{\partial f_{k}(X)}{\partial x_{1 n}} \\
\vdots & & \vdots \\
\frac{\partial f_{1}(X)}{\partial x_{n n}} & \ldots & \frac{\partial f_{k}(X)}{\partial x_{n n}}
\end{array}\right)
$$

Let $\iota_{i j}(M)$ denote the $i j$ th entry of the matrix $M$ and let

$$
J(X):=\left(\begin{array}{cccc}
\iota_{11}(I) & \iota_{11}\left(A^{t}+X^{t}\right) & \ldots & \iota_{11}\left(\left(A^{t}+X^{t}\right)^{n-1}\right) \\
\vdots & \vdots & & \vdots \\
\iota_{1 n}(I) & \iota_{1 n}\left(A^{t}+X^{t}\right) & \ldots & \iota_{1 n}\left(\left(A^{t}+X^{t}\right)^{n-1}\right) \\
\vdots & \vdots & & \vdots \\
\iota_{n n}(I) & \iota_{n n}\left(A^{t}+X^{t}\right) & \ldots & \iota_{n n}\left(\left(A^{t}+X^{t}\right)^{n-1}\right)
\end{array}\right)
$$


Theorem 2.4. Let $\Delta \subset \mathbb{F}\left[x_{11}, \ldots, x_{n n}\right]$ be the ideal generated by the $(m+n) \times$ $(m+n)$ minors of the matrix $[J(X), T(X)]$. Then $\chi$ is not dominant for a particular matrix $A$ if and only if $\Delta \subset I(\mathcal{X})$.

Proof. With respect to the standard basis of $M a t_{n \times n}$ the matrix $T(X)$ defines a linear transformation $T(X): \mathbb{F}^{n^{2}} \rightarrow \mathbb{F}^{k}, x \mapsto x T(X)$ and $\operatorname{ker}(T(X))=T_{X}(\mathcal{X})$. The rank of $T(X)$ is by assumption at most $m$, the dimension of $\mathcal{X}$. Similarly the matrix $J(X)$ defines a linear transformation $J(X): \mathbb{F}^{n^{2}} \rightarrow \mathbb{F}^{n}, x \mapsto x J(X)$ and $J(X)$ restricted to $T_{X}(\mathcal{X})$ is exactly $d \psi_{X}$. The concatenated matrix $[J(X), T(X)]$ induces a linear map $\tau: \mathbb{F}^{n^{2}} \rightarrow \mathbb{F}^{n} \oplus \mathbb{F}^{k}$.

If $\Delta \subset I(\mathcal{X})$, then $\mathcal{X} \subset V(\Delta)$, the algebraic set defined by $\Delta$. It follows that the rank of $\tau$ is strictly less than $m+n$ for all matrices $X \in \mathcal{X}$. It is therefore not possible to find a smooth point $P$ whose associated map $d \psi_{P}$ has full rank $n$. By the dominant morphism theorem $\psi$ and therefore $\chi$ is not dominant.

On the other hand if $\Delta \not \subset I(\mathcal{X})$, then there is a smooth point $P$ such that $[J(P), T(P)]$ has rank $m+n$. Since $T(P)$ has rank $m$ it follows that for every $y \in \mathbb{F}^{n}$ the point $(y, 0) \in \mathbb{F}^{n} \oplus \mathbb{F}^{k}$ is in the image of $\tau$. It follows that $d \psi_{P}$ is surjective and once more by the dominant morphism theorem $\psi$ and $\chi$ are dominant.

The following statement is a reformulation:

Corollary 2.5. $\chi$ is dominant for a particular matrix $A$ if and only if the matrix $[J(X), T(X)]$ has rank $m+n$ over the ring $\mathcal{O}(\mathcal{X})$.

Remark 2.6. Theorem 2.1 did assume that the characteristic of $\mathbb{F}$ is zero. If the characteristic of $\mathbb{F}$ is $p$ and $p>n$, then our proof of Theorem 2.1 is still valid. If $0<$ $p \leq n$, then the Newton identities expressing the elementary symmetric functions $\sigma_{i}(M)$ in terms of the power sum symmetric functions $\left\{\operatorname{tr}\left(M^{j}\right) \mid 1 \leq j \leq n\right\}$ do not exist and our proof method does not go through. It therefore remains an open question if Theorem 2.1 is also true in characteristic $p$, where $0<p \leq n$.

\section{REFERENCES}

[Bor91] A. Borel, Linear algebraic groups, second enlarged edition, Graduate Texts in Mathematics, vol. 126, Springer-Verlag, New York, 1991. MR 92d:20001

[Byr89] C. I. Byrnes, Pole assignment by output feedback, Three Decades of Mathematical System Theory (H. Nijmeijer and J. M. Schumacher, eds.), Lect Notes in Control and Information Sciences \# 135, Springer Verlag, 1989, pp. 31-78. MR 90k:93001

[GKS95] I. Gohberg, M. A. Kaashoek, and F. van Schagen, Partially specified matrices and operators: Classification, completion, applications, Birkhäuser, Boston-Basel-Berlin, 1995. MR 97i: 47002

[HRW97] W. Helton, J. Rosenthal, and X. Wang, Matrix extensions and eigenvalue completions, the generic case, Trans. Amer. Math. Soc. 349 (1997 no. 8, 3401-3408.) MR 97m:15010

[RW97] J. Rosenthal and X. Wang, Inverse eigenvalue problems for multivariabl linear systems, Systems and Control in the Twenty-First Century (Boston-Basel-Berlin) (C. I. Byrnes, B. N. Datta, D. Gilliam, and C. F. Martin, eds.), Birkäuser, Boston-Basel-Berlin, 1997, pp. 289-311. MR 97k:93027

Department of Mathematics, University of Notre Dame, Notre Dame, Indiana 465565683

E-mail address: Rosenthal.1@nd.edu

Department of Mathematics, Texas Tech University, Lubbock, Texas 79409-2013

E-mail address: mdxia@ttacs1.ttu.edu 